Acta Botanica Brasilica - 30(3): 371-382. July-September 2016. doi: 10.1590/0102-33062016abb0026

\title{
A Synopsis of Sloanea [Elaeocarpaceae] in the Neotropical extra-Amazonian Region
}

\author{
Daniela Sampaio ${ }^{1 *}$ and Vinicius Castro Souza ${ }^{2}$
}

Received: January 28, 2016

Accepted: May 31, 2016

\begin{abstract}
The genus Sloanea is comprised of 150 species, of which about 50 occur in Brazil among several vegetation types but mainly the Amazon and Atlantic forests. The present work provides a synopsis of the Neotropical species of Sloanea in the extra-Amazonian region based on a recent revision of the genus. In general, morphologically Sloanea comprises large trees endowed with buttressed roots; simple leaves; flowers with sepals that may or may not cover the reproductive organs in pre-anthesis phases; stamens with the connective prolonged into an awn that is acuminate, acute or aristate and fruit covered with rigid or flexible bristles or sometimes unarmed. This synopsis describes 17 species, and provides an identification key, illustrations and comments on their diagnostic characters, geographical distribution and main bibliographic references.
\end{abstract}

Keywords: Atlantic Forest, Brazil, diversity, savanna, taxonomy

\section{Introduction}

Sloanea, the largest genus of the family Elaeocarpaceae, contains about 150 species distributed in Australia, Asia, Madagascar, South America, Central America and Mexico (Steyermark 1952; 1978; 1988; Smith 1954; 1965; 1967; 1996; Coode 1983; Palacios-Duque 2004; 2005; 2007; Palacios-Duque \& Fernándes-Alonso 2005; Sampaio \& Souza 2010; 2011a; b; Boeira et al. 2012). In Brazil, the genus can be found throughout the country but particularly in more humid and preserved forest formations. The main contributions to the genus for the Neotropics were the taxonomic treatments published by Schumann (1886) and Smith (1954). The latter proposed the division of Sloanea into two subgenera and four sections (Subgenus Sloanea: Sections Sloanea and Brevispicae; Subgenus Quadrisepala: Sections Paniculi and Corymbo-racemi), based on the number of sepals and the position of the calyx in the floral bud, the persistence of the stipules and the type and position of the inflorescence. In this work, the infra-generic classification proposed by Smith (1954) is recognized in part because we consider that the position of the calyx in relation to the bud is a good taxonomic character for the recognition of subgen. Sloanea (calyx not covering essential organs in the bud) and subgen. Quadrisepala (calyx covering the essential organs in the bud). The other characters used by Smith (1954) were not considered because of their overlapping distribution among the two subgenera and the four sections (Sampaio \& Souza 2011a).

This synopsis is derived from a recent revision of the genus for the Neotropical extra-Amazon region (Sampaio 2009) and aims to contribute to the identification of recognized species by providing an identification key and diagnostic morphological characters, illustrations and current geographic distribution.

\footnotetext{
${ }^{1}$ Instituto de Biociências, Letras e Ciências Exatas, Departamento de Zoologia e Botânica, Universidade Estadual Paulista, UNESP, Rua Cristóvão Colombo, 2265, 15054-000, São José do Rio Preto, SP, Brazil

${ }^{2}$ Departamento de Ciências Biológicas, Escola Superior de Agricultura Luis de Queiros, Universidade de São Paulo, Av. Pádua Dias, 11, 13418-900, Piracicaba, SP, Brazil

* Corresponding author: dsampaio@ibilce.unesp.br
} 


\section{Materials and methods}

This work is the result of a review and analysis of collections and nomenclatural types available in 55 Brazilian and foreign herbaria cited throughout the paper, whose acronyms follow Holmgren \& Holmgren (1998). Field expeditions were conducted in the following Brazilian states: Amazonas, Bahia, Espírito Santo, Goiás, Minas Gerais, Rio de Janeiro, Santa Catarina, São Paulo and Paraná. Collected material was deposited in the following herbaria: Escola Superior de Agricultura "Luiz de Queiroz", University of São Paulo (ESA) and University of Campinas (UEC).

Dimensions of vegetative and reproductive structures presented in the descriptions were measured from dried herbarium material (except for floral structures, which were rehydrated prior to measurement). Information concerning habit, flower coloration and other floral structures were obtained from data provided in the labels of herbarium material and, when possible, from in situ observations.

The morphological terminology used for vegetative and reproductive structures was based on Smith (1954), which is the standard for the genus, and Radford et al. (1976). The venation pattern of leaves followed the classification of Hickey (1979), and the terminology used for inflorescences was adapted from Weberling (1989). Abbreviations for the works cited in this study were based on Stafleu \& Cowan (1981), whereas those of authors of genera and species followed Brummitt \& Powell (1992).

\section{Results and discussion}

Sloanea L., Sp. Pl. 1: 512, 1753

=Ablania Aublet, Pl. Guian.1: 585, táb. 234. 1775.

Trees or rarely shrubs. Trunk usually buttressed. Branchlet usually lenticellate. Leaves alternate, opposite or rarely whorled; stipules present, usually caducous; petioles incrassate at one or both ends, terete or canaliculate; leaf-blade with margin entire, crenate, serrate or dentate, with variable shape and size, venation brochidodromus, craspedodromous or eucamptodromous. Inflorescences axillary, rarely terminal, racemose or cymose, rarely solitary. Flowers bisexual; monochlamydeous or dichlamydeous; 4 - 11 sepals, equal or unequal, usually free, valvate; petals usually absent; circa 50 to more than 100 stamens; anthers basifixed, connective prolonged above the anther into an awn; ovary sessile or with stipe, $3-6$ loculi; $8-10$ ovules per locule; style entire or parted; floral receptacle convex. Fruit with loculicidal capsule, variable shape and size; $3-6$ valves, woody, unarmed or armed with flexible or rigid bristles, straight or curved, firmly attached or easily detached, rarely irritant. Seeds 1 - many per capsule, covered up to the mid region or entirely by an aril.

According to Sampaio \& Souza (2011a), the genus Sloanea can be recognized by the size of the tree (2 to 35 meters high), single leaves with swelling at both ends of the petiole, monochlamydeous flowers and fruits covered by flexible bristles. However, as exceptions, some species are of smaller adult size, such as $S$. hirsuta; have dichlamydeous flowers, such as $S$. petalata, or have glabrous and unarmed fruits, such as $S$. floribunda.

Neotropical species of the genus Sloanea are characterized by having simple leaves with alternate or opposite insertions, distributed along the branches or concentrated at the apex. In contrast, Coode (1983) noted that Sloanea sogorensis, an Old World species (New Guinea), has compound young leaves. Several juvenile individuals of species of Sloanea were observed during field expeditions for this work, but none exhibited compound leaves. Another important characteristic of Sloanea sogorensis, is the presence of a pair of stipules lateral to the petiole. In extra-Amazonian species the stipules may fall prematurely, and so these structures can be seen more easily in young branches. Some species have persistent stipules that remain until the maturation of the branches, but they soon fall (S. garckeana, S. lasiocoma, S. hirsuta, S. pubescens, S. retusa, S. terniflora, S. hatschbachii, S. uniflora, S. filiformis and S. fasciculata). In extra-Amazonian species stipules vary in size (from 1.5 - $9 \mathrm{~mm}$ length) and shape (lanceolate, filiform or ovate).

The most important diagnostic characteristics used to differentiate species of Sloanea are the type of inflorescence, the position of the sepals in the bud, the shape of the anther connective extension and, to a lesser extent, the arrangement and structure of the bristles on the capsule.

In the extra-Amazonian region, flowers of Sloanea are monochlamydeous with only the calyx present. They are formed by one or two whorls of sepals (S. lasiocoma) with variable shape and size. The presence of sepals covering (Fig. 1A) or not the reproductive organs in the bud (Fig. 1B) is another useful taxonomic character. Likewise, the anther connective extension is an important character and is used to circumscribe many species of the genus. In this work, the genus was divided into three informal groups (acuminate, aristate and acute) based on the length and shape of the anther connective extension (Fig. 2).

The acuminate extension of $S$. guianensis, Sloanea hatschbachii, S. subsessilis and S. terniflora is about $1 \mathrm{~mm}$ long, and emerges from the anther maintaining constant width towards the apex (Fig. 2A-D). In other species, the extension of the aristate connective varies between 2 and $4 \mathrm{~mm}$ in length and arises abruptly from the anther, tapering towards the apex. Among the species with these characteristics are: S. garckeana, S. filiformis, S. floribunda, S. petalata and S. uniflora (Fig. 2E-I). The acute connective extension is less than or equal to $0.5 \mathrm{~mm}$ in length in S.eichleri, S.fasciculata, S. lasiocoma, S. hirsuta, S. obtusifolia and $S$. retusa (Fig. 2J-O).

The gynoecium also possesses characters useful for the delimitation of species of Sloanea (Fig. 3). The shape, size and, especially, type of indument covering the ovaries are 


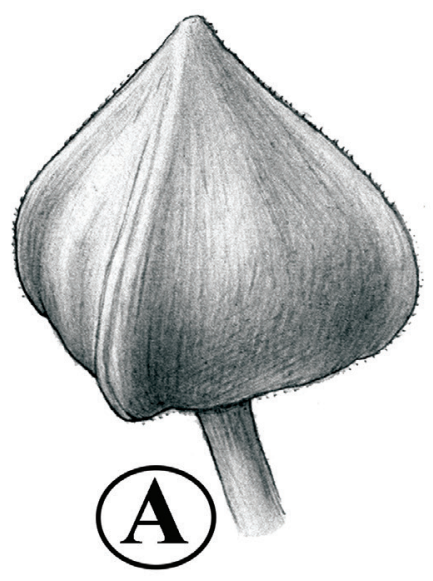

Figure 1. (A) S. lasiocoma flower bud; (B) S. eichleri flower buds.

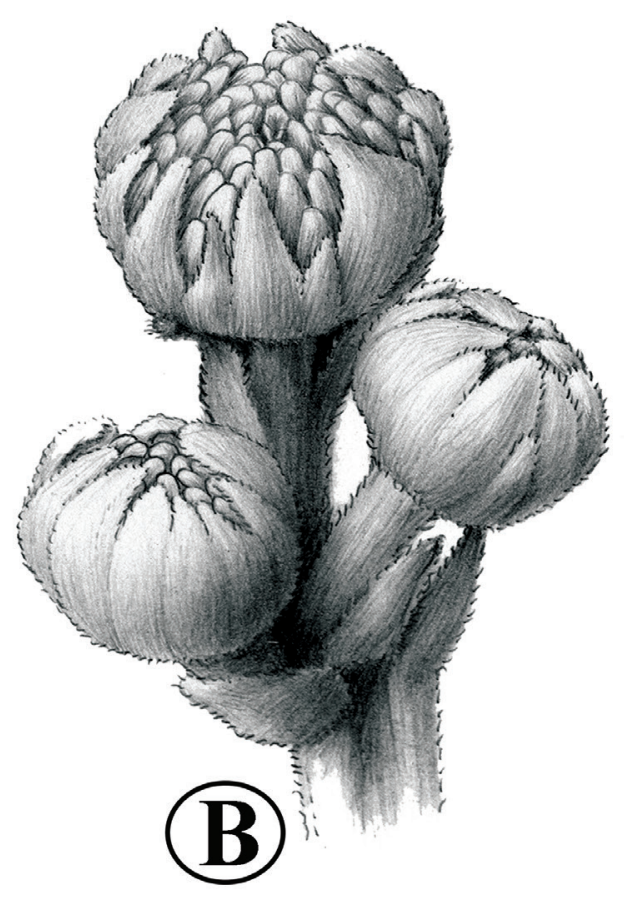

used to separate some groups of species: $S$. garckeana and $S$. petalata, for example, have the ovary covered with a long-velutinous indument with branched hairs (Fig. 3H-I). This type of indument does not occur in other species of the extra-Amazonian region, and although branched hairs are also common in S. uniflora and S. terniflora, the type of indument covering the ovaries in these species is of the short-pubescent type (Fig. 3G, J).

The style, whose length varies between 1.5 and 8 $\mathrm{mm}$, is also important for circumscribing some species of Sloanea. Sloanea eichleri, S. hatschbachii and S. filiformis, for example, have styles of 6 to $8 \mathrm{~mm}$, the largest among extra-Amazonian species (Fig. $3 \mathrm{~K}, \mathrm{~N}-\mathrm{O}$ ). Styles can be straight or twisted; however, since this is a highly variable character, it was not used for diagnosing taxa. The apex of styles can be whole, divided only at the apex or entirely divided throughout their length. In some species (e.g., S. floribunda, S. garckeana, S. petalata, S. terniflora and S. uniflora) the apex of the style is always entire (Fig. 3A, G-J), while in $S$. filiformis the style is deeply divided (Fig. $3 N$ ).

The fruits of species of Sloanea are loculicidal, woody capsules covered by an indument, which varies widely, and ornamented with stiff or flexible bristles, or is unarmed. Although the fruits, in general, are not good diagnostic characters, they may help in the recognition of some species. For example, in $S$. uniflora the bristles overlaying fruits are variable in length and indument, and may be an irritant, whereas in other species, such as $S$. floribunda, bristles may be completely absent.

The 17 species of Sloanea occurring in the extraAmazonian region presented below were studied in the $\mathrm{PhD}$ thesis of the first author (Sampaio 2009). Of these, six were described as new by Sampaio \& Souza (2010; 2011a; 2011b). Information concerning nomenclatural, relationships among taxa and geographical distribution was provided by Sampaio \& Souza (2014). Headings containing nomenclatural types, original works, synonyms and basionyms, as well as descriptions for all species are provided below.

1. Sloanea eichleri K. Schumann, Fl. Bras. 12 (3): 183, pl. 38, Fig. 1. 1886. Lectotype (designated by Sampaio \& Souza 2014: 122): BRAZIL. Rio Tocantins, no date, Weddel 2478 (P!, isolectotype B barcode B 10 0249968!).

=Sloanea obidensis Ducke, Arch. Inst. Biol. Veg. Rio de Janeiro 2 (2): 160. 1935. Lectotype (designated by Sampaio \& Souza 2014: 122): BRAZIL. Pará: Óbidos, 11 November, 1919, Ducke s.n. (RB12369 barcode 538366!, isolectotype $\mathrm{BM} !)$.

Tree, 3 - $30 \mathrm{~m}$ in height. Leaves alternate to sub-opposite; stipules, ca. $2 \times 1 \mathrm{~mm}$, caducous; petioles $1.5-18 \mathrm{~cm}$ long; leaf blade $8.5-40 \times 5.5-23 \mathrm{~cm}$, elliptical or obovate, margin undulate or serrate, rarely entire. Inflorescence axillary or cauliflorous; thyrsoid or botryoid; peduncle $2-6 \mathrm{~cm}$ long. Flowers with 7 - 9 sepals, $1-1.5 \mathrm{x}$ ca. $1 \mathrm{~mm}$, pubescent on both surfaces, triangular, uniseriate, entire, equal, apex acute, margin flat; sepals not covering the reproductive organs in the floral bud prior to anthesis; anthers $1-1.5$ $\mathrm{mm}$ long, elliptical or lanceolate; connective prolonged into an acute awn less than $0.5 \mathrm{~mm}$ long; ovary ca. $2 \mathrm{~mm}$ long, pubescent, globose, sessile; style $6-8 \mathrm{~mm}$ long, twisted or straight, 4 - parted at the apex. Fruits elliptical or globose; 4 valves, $1.5-2 \mathrm{x} \mathrm{ca} .0 .5 \mathrm{~cm}$, covered by bristles $4-15 \mathrm{~mm}$ 


\section{Key to the species of Sloanea of the extra-Amazonian region}

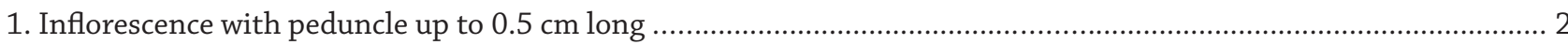

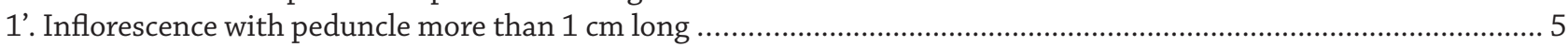

2. Inflorescence fasciculate

2. S. fasciculata

2'. Inflorescence bracteate, racemose .....

3. Sepals with revolute margins and fully covering the reproductive organs in the bud prior to anthesis

3'. Sepals with flat margins and not fully covering the reproductive organs in the bud prior to anthesis

8. S. hirsuta

4. Style $2-2.5 \mathrm{~mm}$ long

4'. Style $6-8 \mathrm{~mm}$ long

11. S. obtusifolia

10. S. obtusa

5. Sepals with flat margins, not fully covering the reproductive organs in the floral bud prior to anthesis, inflorescence axillary or cauliflorous

5'. Sepals with revolute margins, fully covering the reproductive organs in the bud prior to anthesis, inflorescence axillary or terminal

6. Connective prolonged into an aristate awn

3. S. filiformis

6. Connective prolonged into an acuminate or acute awn 7

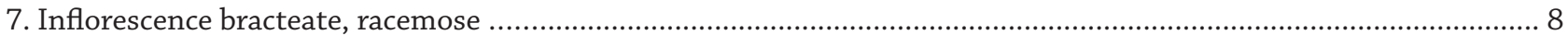

7'. Inflorescence botryoid, or indeterminate thyrse or thyrsoid............................................................................. 9

8. Leaf blade $4.5-18 \mathrm{~cm}$ wide, abaxial surface puberulent to densely-pubescent, venation brochidodromus; peduncle of the inflorescence $2-5 \mathrm{~cm}$ long, ovary $2.5-3 \mathrm{~mm}$ long

13. S. pubescens 8'. Leaf blade $2-3.7 \mathrm{~cm}$ wide, abaxial surface glabrous, venation predominantly craspedodromous; peduncle of the inflorescence $0.1-1.5 \mathrm{~cm}$ long, ovary $1-1.5 \mathrm{~mm}$ long

11. S. obtusifolia

9. Connective prolonged into an acuminate awn

6. S. guianensis

9'. Connective prolonged into an acute awn ... 10

10. Leaf blade up to $3.7 \mathrm{~cm}$ wide, peduncle up to $1.5 \mathrm{~cm}$ long

11. S. obtusifolia

10'. Leaf blade bigger than $5.5 \mathrm{~cm}$ wide, peduncle of the inflorescence bigger than $2 \mathrm{~cm}$ long .. 11

11. Inflorescence thyrsoid; sepals triangular, $1-1.5 \mathrm{~mm}$ long; style $6-8 \mathrm{~mm}$ long; fruits $1.5-2 \mathrm{~cm}$ long

11'. Inflorescence botryoid; sepals ovate or lanceolate, 3 - $4 \mathrm{~mm}$ long; style $2.5-3 \mathrm{~mm}$ long; fruits longer than $2 \mathrm{~cm} . .$.

14. S. retusa

12. Inflorescence triad

13

12'. Inflorescence thyrsoid, or unifloral, or a frondose-bracteate raceme, or a bracteate raceme, or an indeterminate thyrse, or botryoid

13. Petals present

12. S. petalata

13'. Petals absent

14

14. Connective prolonged into an aristate awn $2-4 \mathrm{~mm}$ long; style $3-5 \mathrm{~mm}$ long; ovary with longvelutinous indument

5. S. garckeana

14'. Connective prolonged into an acuminate awn ca. $1 \mathrm{~mm}$ long; style ca. $1.5 \mathrm{~mm}$ long; ovary with pubescent indument

15. Inflorescence unifloral or thyrsoid

16. S. terniflora

15'. Inflorescence a frondose-bracteate raceme, a bracteate raceme, an indeterminate thyrse or botryoid

16. Inflorescence unifloral, ovary sessile covered by branched trichomes

16 '. Inflorescence thyrsoid, ovary stipitate or sub-stipitate, covered by simple trichomes

17. S. uniflora

17. Inflorescence frondose-bracteate raceme, bracteate raceme, or indeterminate tyrse

4. S. floribunda

17'. Inflorescence botryoid

18

18. Connective prolonged into an acuminate awn, style around $7 \mathrm{~mm}$ long

15. S. subsessilis

18. Connective prolonged into an acute awn, style up to $4 \mathrm{~mm}$ long

7. S. hatschbachii

18. Connective prolonged into an acute awn, style up to $4 \mathrm{~mm}$ long ..................................................................... 19

19. Peduncle of the inflorescence $1-4.5 \mathrm{~cm}$ long; $4-6$ (rarely 8) sepals; style $2.5-4 \mathrm{~mm}$ long; inflorescence a bracteate raceme, frondose-bracteate raceme or indeterminate thyrsi

9. S. lasiocoma

19'. Peduncle of the inflorescence $0.2-1 \mathrm{~cm}$ long, 4 (rarely 6) sepals; style $1.5-2.5 \mathrm{~mm}$ long; inflorescence a bracteate raceme 

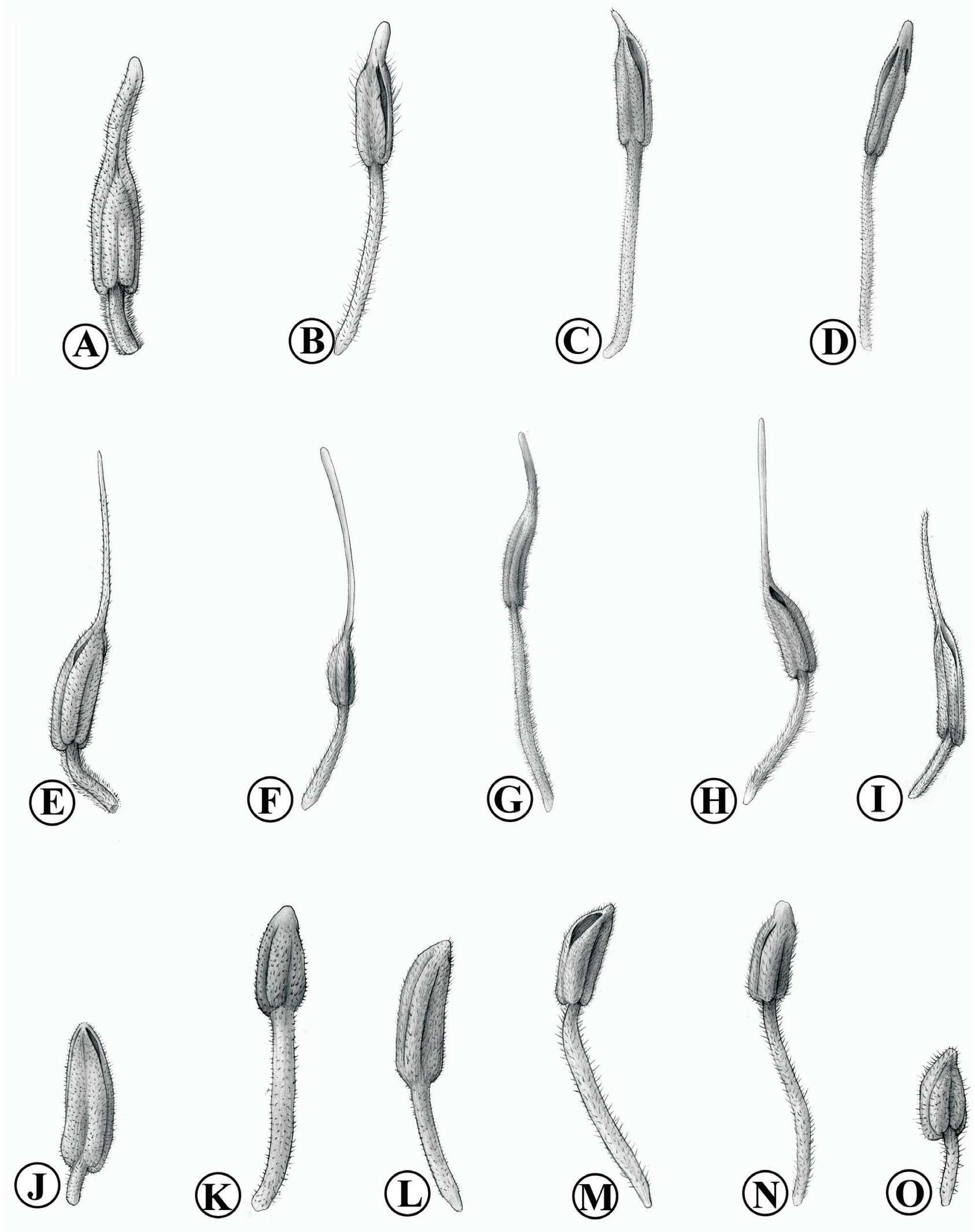

Figure 2. Details of the stamens of extra-Amazonian species of Sloanea. (A-D) Connective prolonged into an acuminate awn. (A) S. terniflora; (B) S. guianensis; (C) S. hatschbachii; (D) S.subsessilis. (E-I) Connective prolonged into an aristate awn. (E) S. garckeana; (F) S. filiformis; (G) S. uniflora; (H) S. petalata; (I) S. floribunda. (J-O) Connective prolonged into an acute awn. (J) S. hirsuta; (K) S. fasciculata; (L) S. lasiocoma; (M) S. retusa; (N) S. eichleri; (0) S. obtusifolia. 


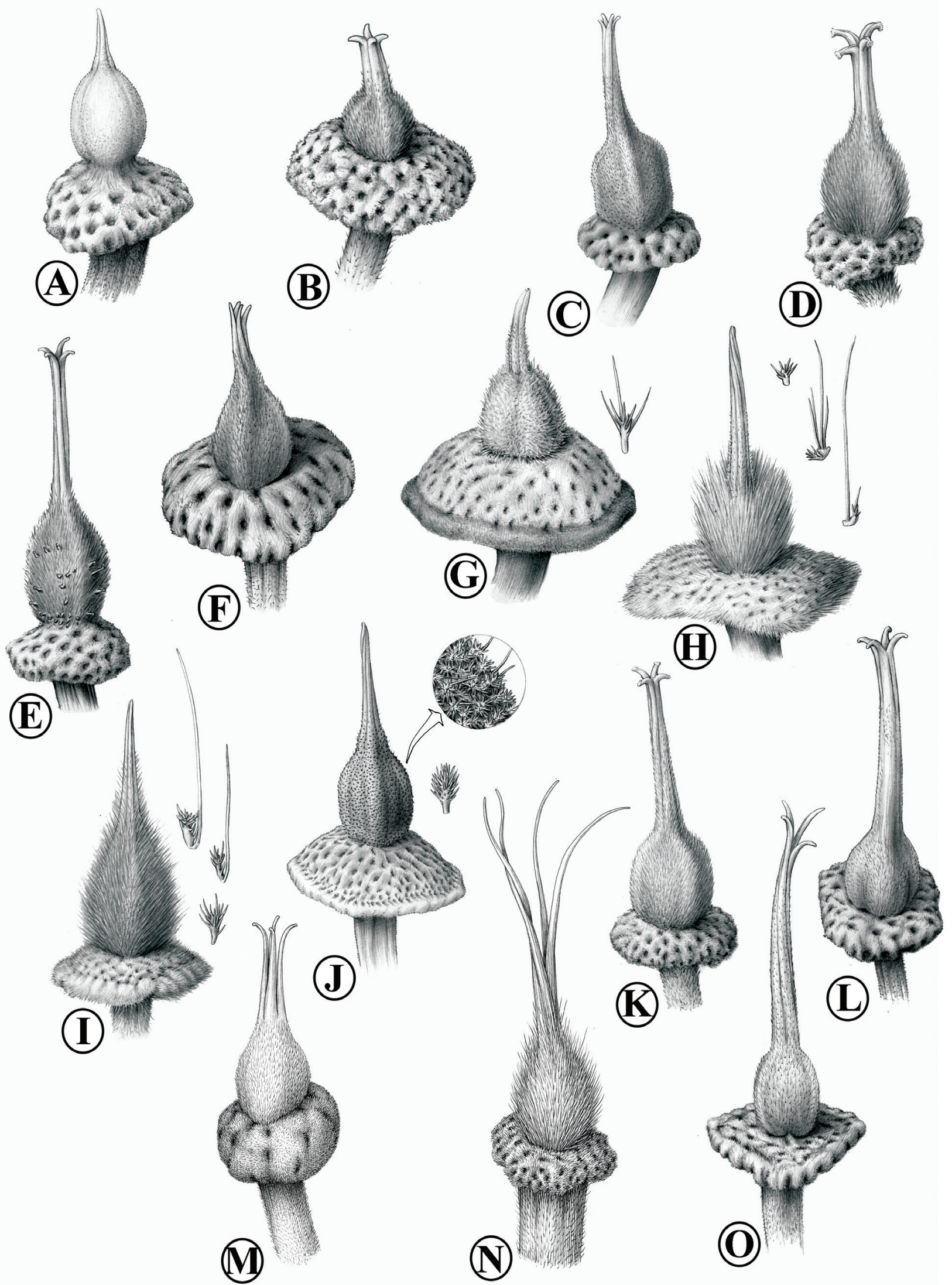

Figure 3. Details of the ovaries of extra-Amazonian species of Sloanea. (A) S. floribunda; (B) S. obtusifolia; (C) S. lasiocoma; (D) S. retusa; (E) S. guianensis; (F) S. hirsuta; (G) S. terniflora; (H) S. garckeana; (I) S. petalata; (J) S. uniflora; (K) S. eichleri; (L) S. subsessilis; (M) S.fasciculata (N) S. filiformis; (O) S. hatschbachii. 
long. Seeds 1, ca. 5 × 3 mm, elliptical, aril not visible.

Geographical distribution: In the extra-Amazonian region this species is found in the Brazilian states of Espírito Santo, Goiás, Mato Grosso and Rio de Janeiro. This species can be seen in Atlantic Forest and gallery forest.

2. Sloanea fasciculata D. Sampaio \& V.C. Souza, Kew Bulletin 66 (4): 512. 2011. Type: Brazil, Espírito Santo, Santa Teresa, L. Kollmann 2166 (holotype ESA!; isotype MBML!).

Tree, 2 - $9 \mathrm{~m}$ in height. Leaves alternate, sub-opposite; stipules persistent, $4-5 \times 0.5 \mathrm{~mm}$; petioles $0.5-1.4 \mathrm{~cm}$ long; leaf-blade $3-7.3 \times 1.5-3.5 \mathrm{~cm}$, elliptical or lanceolate, margin entire. Inflorescences axillary or terminal; fasciculate, sessile or peduncle up to $0.5 \mathrm{~mm}$. Flowers with 4 sepals, $3-3.5 \times 1-1.5 \mathrm{~mm}$, sparsely-pubescent on both surfaces, ovate or lanceolate, uniseriate, entire, equal, apex acute or acuminate, margin planar and white-tomentose on the inner face; sepals covering the reproductive organs in the floral bud near anthesis; anthers ca. $0.5-1 \mathrm{~mm}$ long, elliptical or globose; connective prolonged into an acute awn less than $0.5 \mathrm{~mm}$ long; ovary ca $1-1.5 \mathrm{~mm}$ long, white tomentose, ovate, sessile; style ca $2-2.5 \mathrm{~mm}$ long, straight, 3 - 4 -divided to the base. Fruits ellipsoid; $4-5$ valves, $2-3 \times 0.5-0.7 \mathrm{~cm}$, covered by bristles $2-3 \mathrm{~mm}$ long. Seeds 1 , ca. 10 x ca. 5 mm, elliptical, aril not visible.

Geographical distribution: $S$. fasciculata is an endemic to Brazil and was previously known only from the municipality of Santa Teresa, Espírito Santo State. However, new collections of this species were made in Bahia State. Sloanea fasciculata occurs in the highlands of the Atlantic Forest.

3. Sloanea filiformis D. Sampaio \& V.C. Souza, Phytotaxa 16: 45. 2011. Type: BRAZIL. Bahia: Barreiras, margin of the Rio de Janeiro, gallery forest, 2 November 1987, Queiros 2105 (holotype UEC!, isotypes HUEFS!, K!).

Tree, $4-5 \mathrm{~m}$ in height. Leaves alternate to subopposite; stipules persistent, ca. 5 × $2-3 \mathrm{~mm}$; petioles $1-6 \mathrm{~cm}$ long; leaf blade $8-20 \times 5.5-12 \mathrm{~cm}$, elliptical or obovate, margin finely serrate. Inflorescences axillary or cauliflorous; botryoid; peduncle $1-2.5 \mathrm{~cm}$ long. Flowers with $6-8$ sepals, $3-4 \mathrm{x}$ ca. $1 \mathrm{~mm}$, densely rufous-pubescent on both surfaces, lanceolate, uniseriate, variable in size and shape, apex acuminate, margin planar; not covering the reproductive organs in the floral bud near anthesis; anthers ca. $1-1.5 \mathrm{~mm}$ long, elliptical or ovate; connective prolonged into a long aristate awn $3-3.5 \mathrm{~mm}$ long; ovary ca. $2 \mathrm{~mm}$ long, rufous-tomentose, elliptical, sessile; style ca. 4-6 $\mathrm{mm}$ long, deeply 4 - divided to the base. Fruits elliptical; 4 valves, $1.5-3 \times 0.4-0.5 \mathrm{~cm}$, covered by bristles $3-4 \mathrm{~mm}$ long. Seeds 1 , ca. 20 x ca. $7 \mathrm{~mm}$, elliptical, aril red or orange.

Geographical distribution: Sloanea filiformis occurs in the Brazilian states of Bahia and Mato Grosso, in gallery forest.

4. Sloanea floribunda Spruce ex Bentham, J. Proc. Linn. Soc., Bot. 5 (suppl.2): 66. 1861. Lectotype (designated by Sampaio \& Souza 2014: 122): BRAZIL. San Carlos, Rio
Negro, 1853-54, Spruce 3680 (K barcode 000381954! [Herb. Benth.], isolectotypes BR barcode BR0000006995607!, F barcode V0074597F!, GH barcode 00052202!, K barcode 000381955!, NY barcode 00415327!, P!, RB barcode 00538347!, W barcode 0006499!).

=Sloanea longipes Ducke, Arch. Inst. Biol. Veg. Rio de Janeiro 2 (2): 166. 1935. Lectotype (designated by Sampaio \& Souza 2014: 122): BRAZIL. Pará, Rio Macujubim, 9 July 1923, Ducke s.n., (RB 18379 barcode 00542295!, isolectotypes K!, P!, S!, US barcode 00098572!).

=Sloanea maroana Steyermark, Pittieria 7: 14. 1978. Type: VENEZUELA. Territorio Federal Amazonas, 20 April 1970, Steyermark and Bunting102799 (holotype VEN).

Tree, 8 - $35 \mathrm{~m}$ in height. Leaves alternate; stipules early deciduous, not observed; petioles $1.2-6 \mathrm{~cm}$ long; leaf blade $7.5-20(-23) \times 3.2-7 \mathrm{~cm}$, obovate, rarely lanceolate, margin entire. Inflorescence axillary or terminal; thyrsoid; peduncle $3-7 \mathrm{~cm}$ long. Flowers with 4 sepals, $7-12 \times 3-5$ $\mathrm{mm}$, ovate, rarely lanceolate, uniseriate, entire, equal, apex acuminate; margin revolute and tomentose on the inner surface; covering the reproductive organs in the floral bud near anthesis; anthers $2-4 \mathrm{~mm}$ long, elliptical or lanceolate; connective prolonged into a long aristate awn $2-3 \mathrm{~mm}$ long; ovary 2 - $4 \mathrm{~mm}$ long, short-pubescent, with simple trichomes, globose, stipitate or sub-stipitate, stipe ca. $1 \mathrm{~mm}$ long; style $2-4.5 \mathrm{~mm}$ long, straight or twisted, apex entire. Fruits globose; 4 valves, $2-3 \times 1-1.3 \mathrm{~cm}$, unarmed. Seeds $1,10-15$ x $5-7 \mathrm{~mm}$, aril white in Ducke 1154 .

Geographical distribution: Sloanea floribunda occurs in gallery forests in Mato Grosso State.

5. Sloanea garckeana K. Schumann, Fl. Bras. 12 (3): 177, pl. 36. 1886. Lectotype (designated by Sampaio \& Souza 2014: 123): BRAZIL. Rio de Janeiro, Riedel 888 (K barcode 000382010!, isolectotypes BR barcode BR0000006994969!, L!, M!, P!, W barcode 0003026!, WU barcode 0040322!).

Tree, $2-13 \mathrm{~m}$ in height. Leaves alternate to subopposite; stipules persistent in young branches, $4-8 \mathrm{x}$ ca. $1 \mathrm{~mm}$; petioles 5 - $35 \mathrm{~mm}$ long; leaf blade $5.2-17 \times 2.3$ $5.4 \mathrm{~mm}$, obovate to oblanceolate, margin entire, undulate, rarely serrate in the first upper third of blade. Inflorescences axillary or terminal; triad; peduncle $1-7 \mathrm{~cm}$ long. Flowers with 4 sepals, $5-12 \times 3-7 \mathrm{~mm}$, pubescent on both surfaces, ovate, uniseriate, entire, equal, apex acuminate, margin revolute; sepals covering the reproductive organs in the floral bud near anthesis; anthers $2-3 \mathrm{~mm}$ long, elliptical or lanceolate; connective prolonged into an aristate awn 2 - $4 \mathrm{~mm}$ long; ovary $3-4 \mathrm{~mm}$ long, long-velutinuous with branched trichomes, globose, sessile; style 3 - $5 \mathrm{~mm}$ long, twisted, apex entire. Fruits elliptical or globose; 4 valves $1.5-2.8 \times 0.5-1.3 \mathrm{~cm}$, covered by bristles $3-5 \mathrm{~mm}$ long. Seeds $1,13-18$ x $6-10 \mathrm{~mm}$, elliptical, aril red.

Geographical distribution: Sloanea garckeana has been found in areas of Atlantic Forest and gallery forest in almost all Brazilian states except in Rio Grande do Sul. 
6. Sloanea guianensis (Aublet) Bentham, J. Proc. Linn. Soc., Bot. 5 (suppl. 2): 69. 1861. Ablania guianensis Aublet, Hist. Pl. Guiane 1: 585, t. 234. 1775. Lectotype (designated by Sampaio \& Souza 2014: 123): FRENCH GUIANA. Habitat in sylvis "Sinemarientibus", no date, Aublet s.n. (BM barcode 000795143!).

=Dasynema pubescens Schott, Syst. Veg. 4: Cur. Post. 408. 1827. Type: Onsite, unknown collector (holotype W barcode 0016332!).

=Sloanea alnifolia Martius, Flora 20 (2) Beibl. 94.1837. Dasynema alnifolium (Martius) Walpers, Repert. Bot. Syst. 1: 352. 1842. Lectotype (designated by Sampaio \& Souza 2014: 123): BRAZIL. Rio de Janeiro: Copacabana, December 1853, Martti Herb. Fl. Br 87(BR barcode BR0000006995294!, isolectotype M!).

=Sloanea cuneifolia Martius, Flora 20 (2) Beibl. 94.1837. Dasynema cuneifolium (Martius) Walpers, Repert. Bot. Syst. 1: 352. 1842. Type: BRAZIL. Amazonas. (holotype M!).

=Sloanea microcarpa Planchon ex Bentham, J. Proc. Linn. Soc., Bot. 5 (suppl.2): 69. 1861. Sloanea guianensis var. microcarpa (Planchon ex Bentham) Schumann, Fl. Bras. 12 (3): 191. 1886. Type: VENEZUELA. Cayenne, Martin s.n. (holotype K! [Herb. Hook.]).

=Sloanea alnifolia var. lancea K. Schumann, Fl. Bras. 12 (3): 194. 1886. Lectotype (designated by Sampaio \& Souza 2014: 124): BRAZIL. Rio de Janeiro: September, 1832, Riedel 887 (BR barcode BR0000006995287!, isolectotypes K barcode 000381907!, M!, P!, S!, W barcode 0003024!).

=Sloanea alnifolia var. ovalis K. Schumann, Fl. Bras. 12 (3): 194. 1886. Lectotype (designated by Sampaio \& Souza 2014: 124): BRAZIL. São Paulo: Santos, Mosén 2785 (S 07-12928!, isolectotype S!).

=Sloanea maximowicziana K. Schumann, Fl. Bras. 12 (3): 192. 1886. Lectotype (designated by Sampaio \& Souza 2014: 124): BRAZIL. Una, 1821, Riedel 194 (W barcode 0050844!).

=Sloanea breviseta Steyermark, Fieldiana: Bot. 28: 357 . 1952. Type: VENEZUELA. 19 April, 1945, Steyermark 62215 (holotype F barcode V0074602F!, isotypes NY barcode 00415315 !, US barcode 00098544!).

Tree, $4-25 \mathrm{~m}$ in height. Leaves alternate, sub-opposite or rarely alternate; stipules early deciduous, $1.5-3 \times 0.5-1$ $\mathrm{mm}$; petioles $1.5-7 \mathrm{~cm}$ long; leaf blade $(-2.5) 3.5-28(-31)$ x $3.5-11 \mathrm{~cm}$, obovate, rarely oblanceolate, margin entire, undulate or serrate. Inflorescences axillary or cauliflorous; botryoid; peduncle $1-6 \mathrm{~cm}$ long. Flowers with $6-9(-4)$ sepals, $1.5-5 \times 0.5-2.5 \mathrm{~mm}$, pubescent on both surfaces, ovate or lanceolate, uniseriate, indented, unequal, apex acute or acuminate, margin planar; sepals not covering the reproductive organs in the floral bud near anthesis; anthers 1 $-2.5 \mathrm{~mm}$ long, elliptical or lanceolate; connective prolonged into an acuminate awn ca. $1 \mathrm{~mm}$ long; ovary $2-3 \mathrm{~mm}$ long, pubescent, globose or elliptical, sessile to stipitate, stipe $0.5-1 \mathrm{~mm}$ long; style $3-7 \mathrm{~mm}$ long, twisted or straight, 4 - parted near the apex. Fruits globose or elliptical; 4 valves $1-1.7 \times 0.5-1 \mathrm{~cm}$, covered by bristles $5-20 \mathrm{~mm}$ long. Seeds 1 - many, elliptical, laminar or angular, 2 - 10 x $0.5-5 \mathrm{~mm}$, aril red or orange.

Geographical distribution: S. guianensis occurs in all extra-Amazonian region, in the Atlantic Forest and gallery forests.

7. Sloanea hatschbachii D. Sampaio \& V.C. Souza. Kew Bulletin 66 (4): 512. 2011. Type: Brazil, Paraná, Paranaguá, G. Hatschbach 20091 (holotype MBM!).

Tree, $2.5-4 \mathrm{~m}$ in height. Leaves opposite to alternate; stipules persistent in the young branches, ca. $3 \times$ ca. $0.5 \mathrm{~mm}$; petioles $0.4-1 \mathrm{~cm}$ long; leaf-blade $3-5.7 \times 1.4-3.4 \mathrm{~cm}$, elliptical to obovate, margin entire. Inflorescences axillary or terminal; bracteate racemose or frondose-bracteate racemose; peduncle $1.5-4 \mathrm{~cm}$ long. Flowers with 4 sepals, $7-10 \times 3-5 \mathrm{~mm}$, pubescent on outer surface, densely pubescent on inner surface, ovate, uniseriate, entire, equal, apex acute or acuminate, margin finely revolute and whitetomentose on inner surface; sepals covering the reproductive organs in the floral bud near anthesis; anthers $2-3 \mathrm{~mm}$ long, lanceolate; connective prolonged into an acuminate awn ca. $0.5 \mathrm{~mm}$ long; ovary ca $3 \mathrm{~mm}$ long, pubescent, orbicular, sessile; style ca. $7 \mathrm{~mm}$ long, straight, 4 - divided at the apex. Fruits globose; valves $4,1.5-2 \times 0.7-1 \mathrm{~cm}$, covered by bristles ca. $5 \mathrm{~mm}$ long. Seeds not seen.

Geographical distribution: Sloanea hatschbachii is endemic to Brazilian Atlantic Forest. It is known only from the type locality in the municipality of Paranaguá in the state of Paraná.

8. Sloanea hirsuta (Schott) Planch. ex Bentham, J. Proc. Linn. Soc., Bot. 5 (suppl.2): 70. 1861. Dasynema hirsutum Schott, Syst. Veg. 4: Cur. Post. 408. 1827. Type: BRAZIL. Rio de Janeiro: Tijuca, Gardner 5374 (holotype K! [Herb. Benth.], isotypes K [Herb. Hook.], W barcode 0005525!).

=Sloanea monosperma Vellozo, Fl. Flumin. 225. 1829 (1825). Lectotype (designated by Sampaio \& Souza 2014: 125): BRAZIL. Rio de Janeiro: "Habitat silvis maritimis Regii Praedii Santae Crucis" (no existing specimen), Vellozo, Flora Fluminensis, ic. 5: Fig. 100. 1831 (1827).

=Adenobasium salicifolium Presl, Symb. Bot. 1 (3): 40, t. 27. 1832. Dasynema salicifolium (Presl.) Endlcher ex Walpers, Repert. Bot. Syst. 1(2): 352. 1842. Type: "America Meridionali", J. S. Presl Herb., collector not indicated (holotype PR!).

=Dasynema riparia Gardner, Lond. London J. Bot. 2: 334 . 1843. Sloanea riparia (Gardner) Planchon ex Bentham, J. Proc. Linn. Soc., Bot. 5 (suppl.2): 70. 1861. Type: BRAZIL. Rio de Janeiro: Serra dos Órgãos, Gardner 327 (holotype K! [Herb. Hook.], isotypes G!, K!, NY barcode 00415217!, P!, S!, W barcode 0016333!).

=Sloanea pulverulenta Radlkofer, Sitzungsber. Math.Phys. Akad. Muench. 12: 329. 1882. Sloanea monosperma var. pulverulenta (Radlkofer) K. Schumann, Fl. Bras. 12 (3): 186. 1886. Type: BRAZIL. Rio de Janeiro: Corcovado, Martius s.n. (holotype M!).

=Sloanea monosperma var. hirsutissima K. Schumann, Fl. 
Bras. 12 (3): 186. 1886. Lectotype (designated by Sampaio \& Souza 2014: 125): BRAZIL. Martii Herb. Fl. Bras. 831ex p. (Heb. Brux.) (BR!).

=Sloanea monosperma var. virgata K. Schumann, Fl. Bras. 12 (3): 186. 1886. Lectotype (designated by Sampaio \& Souza 2014: 125): BRAZIL. Rio de Janeiro, Riedel 368 a (BR barcode BR0000006997571!, isolectotypes K barcode 000382006!, M!, P!).

=Sloanea monosperma var. ovalis K. Schumann, Fl. Bras. 12 (3): 186. 1886. Lectotype (designated by Sampaio \& Souza 2014: 125): BRAZIL. São Paulo: Santos, Mosén 2784 (S!).

Tree, 1.5 - $20 \mathrm{~m}$ in height. Leaves alternate to opposite; stipules persistent in the young branches, $2-7 \times 0.5-1$ $\mathrm{mm}$; petioles $0.5-3 \mathrm{~cm}$ long; leaf blade $(-1) 5-12(-16,5)$ $\mathrm{x}(-1.3) 2-5 \mathrm{~cm}$, obovate, oblanceolate, rarely elliptical, margin entire or serrate in the first upper third of blade. Inflorescences axillary; bracteate racemose; peduncle $0.2-1 \mathrm{~cm}$ long. Flowers with 4 (-6) sepals, $3-5 \times 2-4$ $\mathrm{mm}$, pubescent on both surfaces, ovate, rarely lanceolate, uniseriate, equal, entire, apex acute or acuminate, margin finely revolute and white-tomentose on inner surface; sepals covering the reproductive organs in the floral bud near anthesis; anthers $1.5-2.5 \mathrm{~mm}$ long, elliptical; connective prolonged into an acute awn less than $0.5 \mathrm{~mm}$ long; ovary $1.5-3 \mathrm{~mm}$ long, pubescent, globose, sessile; style 1.5 - 2.5 mm long, twisted or straight, $4-5(-3)$ parted at the apex or deeply parted up to the base. Fruits elliptical; 4 valves, rarely $3-5,1.5-3.5 \times 0.4-1.3 \mathrm{~cm}$, covered by bristles $1-4$ $\mathrm{mm}$ long. Seeds 1 , elliptical or obovate, $6-15 \times 4-5 \mathrm{~mm}$, aril orange or red.

Geographical distribution: Sloanea hirsuta occurs only in the Atlantic Forest from Santa Catarina State to Bahia State.

9. Sloanea lasiocoma Schumann, Fl. Bras. 12 (3): 184. 1886. Lectotype (designed by Keller et al. 2012: 158): BRAZIL. Minas Gerais: Caldas, Pedro Branco, Regnell III 1536 (S 07-12933!, isolectotypes BR barcode BR0000006994921!, K barcode 000381995!, M!, NY barcode 00415334!, P!).

=Sloanea monosperma var. coriacea Schumann, Fl. Bras. 12 (3): 186. 1886. Lectotype (designed by Keller et al. 2012: 158):-BRAZIL. Location not indicated, Sello s.n. [233?]: (S!, isolectotypes F barcode V0055207F!, P!).

Tree, 5 - $26 \mathrm{~m}$ in height. Leaves alternate, sub-opposite and whorled at the apex of the branches; stipules persistent or deciduous, $2-4 \mathrm{x}$ ca. $0.5 \mathrm{~mm}$; petioles $(-0.4) 1-3.2 \mathrm{~cm}$ long; leaf blade $2.3-15 \times 0.8-5 \mathrm{~cm}$, obovate, oblanceolate, elliptical or narrow elliptical, margin entire. Inflorescences axillary or teminal; bracteate racemose or frondose-bracteate racemose or indeterminate thyrse; peduncle $1-4.5 \mathrm{~cm}$ long. Flowers with $4-6(-8)$ sepals, $3-5 \times 2-4 \mathrm{~mm}$, puberulous to pubescent on both surfaces, ovate or lanceolate, uniseriate or biseriate, equal, entire, apex acuminate, margin revolute; sepals covering the reproductive organs in the floral bud near anthesis; anthers $2-3 \mathrm{~mm}$ long, elliptical; connective prolonged into an acute awn ca. $0.5 \mathrm{~mm}$ long; ovary 1.5 - 2 $\mathrm{mm}$ long, densely pubescent, ovate, sessile; style $2.5-4$ $\mathrm{mm}$ long, twisted or straight, 4 - parted at the apex or 4 - deeply parted up to the base. Fruits elliptical or globose; $3-4$ valves, $1-2 \times 0.5-1 \mathrm{~cm}$, covered by bristles, generally deciduous, $1.5-8 \mathrm{~mm}$ long. Seeds 1, elliptical or obovate, $8-10 \times$ ca. $5 \mathrm{~mm}$, aril orange or vinaceous.

Geographical distribution: Sloanea lasiocoma occurs in Brazil and Argentina. In Brazil, the species has been collected in the Atlantic Forest in the states of Rio Grande do Sul, Santa Catarina, Paraná, São Paulo and southern Minas Gerais states and a few isolated points in Goiás State. In Argentina, it was collected in the province of Misiones, in a fragment of native forest.

10. Sloanea obtusa (Splitgerber) K. Schumann, Fl. Bras. 12 (3): 181. 1886. Dasymena obtusum Splitgerber, Tijdschr. Nat. Geschied. 9: 98. 1842. Type: SURINAME. Post Belair: April, 1838, Splitgerber 812 (holotype L!).

$=$ Sloanea kappleriana Pulle, Enum. Vasc. Pl. Surinam 279. t. 12. 1906. Lectotype (designated by Sampaio \& Souza 2014: 126): SURINAME. Kapller 2036 (U!, isolectotypes L!, P!, W barcode 0016324!).

Tree, $6-20 \mathrm{~m}$ in height. Leaves opposite to subopposite, rarely alternate; stipules deciduous, not observed; petioles $0.8-2.5 \mathrm{~cm}$ long; leaf-blade $6-14 \times 2.5-7 \mathrm{~cm}$, obovate, margin serrate or crenate. Inflorescences axillary; bracteate racemose; peduncle $0.1-0.5 \mathrm{~cm}$ length. Flowers with $4-5$ sepals, $1-2 \mathrm{x}$ ca. $1 \mathrm{~mm}$, pubescent ferrugineous on both surfaces, ovate, uniseriate, entire or rarely indented, equal, apex acute, margin planar; sepals not covering the reproductive organs in the floral bud near anthesis; anthers ca. $1 \mathrm{~mm}$ long, ovate; connective prolonged into an acute awn less than $0.5 \mathrm{~mm}$ long; ovary ca. $2 \mathrm{~mm}$ long, densely pubescent ferrugineous, elliptical, sessile; style $6-8 \mathrm{~mm}$ long, straight or twisted, 4 - divided at the apex. Fruits elliptical, rarely globose; 4 valves, $1-2.2 \times 0.5-0.7 \mathrm{~cm}$, covered by bristles, $15-20 \mathrm{~mm}$ long. Seeds not seen.

Geographical distribution: Sloanea obtusa occurs in gallery forests in the Brazilian states of Ceará, Mato Grosso and Mato Grosso do Sul.

11. Sloanea obtusifolia (Moric.) K. Schum., Fl. Bras. 12 (3):181. 1886. Adenobasium obtusifolium Moric., Pl. Nouv. Amer. 83, t. 55. 1840. Type: Brasil. Bahia: Blanchet 1659 (holotype: P!; isotype: K!).

=Sloanea fernando-costae Hoehne, Arq. Bot. Estado São Paulo 1: 93, t. 96. 1942. Tipo: Brasil. São Paulo: Jardim Botânico de São Paulo, 21 de Agosto de 1933, SP 30.844, Oswaldo Handro s.n. (holotype: SP!; isotype: GH!, K!, RB!).

Tree, $19-35 \mathrm{~m}$ in height. Leaves alternate; stipules early deciduous, $3-5 \times 1-1.5 \mathrm{~mm}$; petioles $0.4-1.5 \mathrm{~cm}$ long; leaf blade $3.3-9 \times 2-3.7 \mathrm{~cm}$, obovate, rarely elliptical, margin entire, undulate, rarely serrate. Inflorescences axillary or cauliflorous; bracteate racemose or indeterminate 
thyrse; peduncle $0.1-1.5 \mathrm{~cm}$ long. Flowers with $4-5(-8)$ sepals, $2-3 \times 1-2 \mathrm{~mm}$, pubescent on both surfaces, ovate, uniseriate or biseriate, entire or indented, equal or unequal, apex acuminate, margin planar; sepals not covering the reproductive organs in the floral bud near anthesis; anthers ca. $1 \mathrm{~mm}$ long, ovate or globose; connective prolonged into an acute awn ca. $0.5 \mathrm{~mm}$ long; ovary $1-1.5 \mathrm{~mm}$ long, densely pubescent, globose, sessile; style $2-2.5 \mathrm{~mm}$ long, straight, $4-5$ - parted at the apex. Fruits elliptical; $3-4$ valves, $2-3 \times 0.7-1.2 \mathrm{~cm}$, covered by bristles, generally deciduous, $4-10 \mathrm{~mm}$ long. Seeds 1, elliptical, ca. $15 \mathrm{x}$ ca. $7 \mathrm{~mm}$, aril orange.

Geographical distribution: Sloanea obtusifolia occurs in Atlantic Forest in the Brazilian states of Pernambuco to São Paulo.

12. Sloanea petalata D. Sampaio \& V.C. Souza, Rodriguesia 61 (1):13. 2010. Type: BRAZIL. SÃO PAULO: Rodovia Campinas-Mogi Mirim, Auto Posto Varanda, 12.XII.1980, H.Leitão-Filho 12087 (holotype UEC!; isotype RB!).

Trees, $10-15 \mathrm{~m}$ in height. Leaves alternate; stipules early deciduous, $6-9 \times \mathrm{ca} .1 \mathrm{~mm}$; petioles $0.9-1.3 \mathrm{~cm}$ long; leaf blade $7-13 \times 3.5-6 \mathrm{~cm}$, obovate, margin entire or serrate on the first upper third of blade. Inflorescence axillary; triad; peduncle $1.5-4.5 \mathrm{~cm}$ long. Flowers with 4 - sepals, $11-15 \times 3-4 \mathrm{~mm}$, pubescent on both surfaces, ovate, uniseriate, entire, apex acuminate, margin revolute; 4 petals, $7-13 \mathrm{x}$ ca. $1 \mathrm{~mm}$, pubescent on both surfaces, lanceolate, uniseriate, entire, apex acuminate, margin revolute; petals covering the reproductive organs in the floral bud near anthesis; anthers ca. $2 \mathrm{~mm}$ long, elliptical; connective prolonged into an aristate awn $3-4 \mathrm{~mm}$ long; ovary ca. $6 \mathrm{~mm}$ long, velutinous, with branched trichomes, globose, sessile; style ca. $5 \mathrm{~mm}$ long, straight or twisted, apex entire. Fruits orbicular; $2-3 \times 0.7-1.3 \mathrm{~cm}, 4-5$ valves, covered by bristles $2-4 \mathrm{~mm}$ long. Seeds not seen.

Geographical distribution: Sloanea petalata is known only from the type locality, the city of Mogi Guaçu in the state of São Paulo, in an area with predominantly of Cerrado vegetation.

13. Sloanea pubescens Bentham, J. Proc. Linn. Soc., Bot. 5 (suppl.2): 69. 1861. Dasynema pubescens Poeppig and Endlicher (1845: 74)—nom. illeg., non Schott (1827). Lectotype (designated by Sampaio \& Souza 2014: 127): BRAZIL. Amazonas: Ega, Poeppig 2662 (W barcode 0016318!, isolectotypes W barcode 0016320!, GH barcode 00052220!, F barcode V0074610F!).

=Sloanea gracilis Uittien, Recueil Trav. Bot. Néerl. 22: 356. 1925. Type: SURINAME. Browsberg, Bureau Forest, Herb. Numb. 6226. (holotype U!, isotypes K!, L!, RB barcode 00538348!, U!, US!).

=Sloanea xylocarpa Rusby, Mem. New York Bot. Gard. 7: 294. 1927. Type: BOLIVIA. Tumpassa: M. Cardenas 1979 (holotype NY barcode 00415363!, isotypes K!, US barcode 00098605!, GH barcode 00052230!).
=Sloanea pseudodentata Ducke, Arq. Inst. Biol. Veg. 2 (2): 159. 1935. Lectotype (designated by Sampaio \& Souza 2014: 127): BRAZIL. Pará: Rio Tapajós, Ducke s.n. (RB 18378 barcode 00538376!, isolectotype K barcode 000381969!).

Tree, 2 - $22 \mathrm{~m}$ in height. Leaves alternate to subopposite; stipules persistent, $5-6 \mathrm{x}$ ca. $1 \mathrm{~mm}$; petioles $1.5-4 \mathrm{~cm}$ long; leaf blade $7.5-31 \times 4.5-18 \mathrm{~cm}$, obovate or elliptical, margin entire or undulate. Inflorescences axillary or cauliflorous; bracteate racemose; peduncle 2 $-5 \mathrm{~cm}$ long. Flowers with $4-6$ sepals, $3-6 \times 1-2 \mathrm{~mm}$, pubescent on both surfaces, ovate or lanceolate, uniseriate, unequal, indented, apex acuminate, margin planar; sepals not covering the reproductive organs in the floral bud near anthesis; anthers $1-1.5 \mathrm{~mm}$ long, elliptical, theca velutinuous; connective prolonged into an acuminate awn $0.5-1 \mathrm{~mm}$ long; ovary $2.5-3 \mathrm{~mm}$ long, globose, sessile; style $3-4 \mathrm{~mm}$ long, twisted or straight, 4 - parted at the apex. Fruits globose or ovate; $3-4$ valves, $1.5-2 \mathrm{x}$ ca. 1 $\mathrm{cm}$, covered by bristles $15-25 \mathrm{~mm}$ long. Seeds 1 , planar, $5-7 \mathrm{~mm}$ long, ca. $3 \mathrm{~mm}$ wide, aril not seen.

Geographical distribution: Sloanea pubescens can be found in the Atlantic Forest and in gallery forest of the following Brazilian states: Alagoas, Bahia, Minas Gerais, Pernambuco, Goiás and Mato Grosso.

14. Sloanea retusa Uittien, Recueil Trav. Bot. Néerl. 22: 353. 1925. Type: BRAZIL. Rio de Janeiro: Rio de Janeiro, Glaziou 10332 (holotype U!, isotypes K barcode 000381984!, P!).

Tree, 10 - $25 \mathrm{~m}$ in height. Leaves opposite to subopposite, rarely alternate; stipules persistent in young branches, $4-6 \mathrm{x} \mathrm{ca} .1 \mathrm{~mm}$; petioles $1.2-3.5 \mathrm{~cm}$ long; leaf blade $8-17.5 \times 6-8 \mathrm{~cm}$, obovate, margin sparsely serrate, rarely undulate. Inflorescences axillary; botryoid; peduncle $2-10 \mathrm{~cm}$ long. Flowers with $8-9$ sepals, $3-4 \mathrm{x}$ $1-1.5 \mathrm{~mm}$, tomentose on both surfaces, ovate or lanceolate, uniseriate, unequal, entire or indented, apex acute, margin planar; sepals not covering the reproductive organs in the floral bud near anthesis; anthers ca. $1.5 \mathrm{~mm}$ long, elliptical; connective prolonged into an acute awn ca. $0.5 \mathrm{~mm}$ long; ovary $2-2.5 \mathrm{~mm}$ long, velutinuous, ovate, sessile; style $2.5-3 \mathrm{~mm}$ long, straight, 4 - parted at the apex. Fruits elliptical; $4-5$ valves, $2.3-3.2 \times 0.7-1 \mathrm{~cm}$, covered by bristles $1.5-2.5 \mathrm{~mm}$ long. Seeds 1 , elliptical, ca. 15 x ca. $1 \mathrm{~mm}$, aril red.

Geographical distribution: Sloanea retusa occurs in the Atlantic Forest of the Brazilian states of Rio de Janeiro, Espírito Santo, Minas Gerais, Alagoas and Bahia.

15. Sloanea subsessilis D. Sampaio \& V.C. Souza. Phytotaxa 16: 47. 2011. Type: BRAZIL. Mato Grosso: Barra do Bugres, Fazenda Ochsenfeld, mata de galeria, 23 October 1995, Hatschbach et al. 63790 (holotype MBM!).

Tree, 6 - $8 \mathrm{~m}$ in height. Leaves opposite to alternate; stipules early deciduous, not observed; petioles $2-4 \mathrm{~mm}$ 
long; leaf blade $7-23 \times 3.5-11 \mathrm{~cm}$, elliptical or obovate, margin entire. Inflorescences axillary or terminal; botryoid; peduncle $1-5 \mathrm{~cm}$ long. Flowers with 4 sepals, $5-8 \times 2-4$ $\mathrm{mm}$, densely pubescent to tomentose on both surfaces, lanceolate, rarely ovate, uniseriate, equal, entire, apex acuminate, margin revolute; covering the reproductive organs in the floral bud near anthesis; anthers $2-3 \mathrm{~mm}$ long, oblong; connective prolonged into a short acuminate awn ca. $1 \mathrm{~mm}$ long; ovary ca. $2 \mathrm{~mm}$ long, short-pubescent, globose, sessile; style ca. $5 \mathrm{~mm}$ long, straight, 4 - parted at the apex. Fruits not seen.

Geographical distribution: Sloanea subsessilis occurs in gallery forest in the Brazilian state of Mato Grosso.

16. Sloanea terniflora (DC.) Standley, Trop. Woods 79: 10. 1944. Lecostemon terniflorum De Candolle (1825: 639). Lectotype (designed by McVaugh 2000: 218): MEXICO. Location not indicated, collector not indicated (no existing specimen: Tab. "The Torner Collection, n. 1944").

=Sloanea quadrivalvis Seemann, Bot. Voy. Herald: 85, t. 15. 1853. Dasycarpus quadrivalvis (Seemann) Oersted, Vidensk. Meddel. Dansk Naturhist. Foren. Kjobenhavn 1855: 27. 1857. Type: PANAMA. Veraguas, 1849, Seemann s.n. (holotype K!, isotype K!).

=Sloanea reticulata A. C. Smith, Bull. Torrey Bot. Club 61: 194. 1934. Type: BRAZIL. Maranhão: 16 September 1932, Froés 1918 (holotype NY barcode 00415350!, isotypes F barcode V0055211F!, G!, GH barcode 00052222!, K barcode 000382009!, P!, S!).

Tree, $7-12 \mathrm{~m}$ in height. Leaves alternate; stipules persistent in young branches, $3-5 \mathrm{x}$ ca. $0.5 \mathrm{~mm}$; petioles 2 - $10 \mathrm{~mm}$ long; leaf blade $4.5-9 \times 2.3-4.5 \mathrm{~cm}$, obovate, margin entire or serrate in first upper third of blade. Inflorescences axillary; triad; peduncle $1.5-3 \mathrm{~cm}$ long. Flowers with 4 sepals, $4-6 \times 3-4 \mathrm{~mm}$, pubescent on both surfaces, ovate, uniseriate, entire, equal, apex acuminate, margin revolute; sepals covering the reproductive organs in the floral bud near anthesis; anthers $1-1.5 \mathrm{~mm}$ long, elliptical; connective prolonged into an acuminate awn ca. $1 \mathrm{~mm}$ long; ovary ca. $1.5 \mathrm{~mm}$ long, short-pubescent, ovate, with branched trichomes, sessile; style ca. $1.5 \mathrm{~mm}$ long, twisted, apex entire. Fruits elliptical or globose; $4-5$ valves, $1-1.5 \times 0.5 \mathrm{~cm}$, covered by bristles ca. $1 \mathrm{~mm}$ long. Seeds 1, ovate, ca. 8 x ca. $4 \mathrm{~mm}$, aril not seen.

Geographical distribution: Sloanea terniflora has been recorded along rivers, wetlands and floodplains in the Brazilian states of Bahia, Maranhão, Mato Grosso, Paraná and São Paulo.

17. Sloanea uniflora D. Sampaio \& V.C. Souza. Phytotaxa 16: 49. 2011. Type: BRAZIL. Mato Grosso: São Félix, beira do Rio das Mortes, 17 March 1997, Souza et al. 14379 (holotype ESA!, isotype SPSF!).

Tree, $2-12 \mathrm{~m}$ in height. Leaves alternate to subopposite; stipules persistent, $2.5-5 \mathrm{x}$ ca. $1 \mathrm{~mm}$; petioles
$1-3 \mathrm{~cm}$ long; leaf blade $5.5-16.3 \times 2.5-7.7 \mathrm{~cm}$, elliptical to obovate, margin entire or undulate. Inflorescences axillary; unifloral; peduncle $2-4.3 \mathrm{~cm}$ long. Flowers with 4 sepals, $9-12 \times 5-7 \mathrm{~mm}$, tomentose on both surfaces, ovate, uniseriate, entire, equal, apex acuminate, margin revolute and white-velutinous on inner surface; sepals covering the reproductive organs in the floral bud near anthesis; anthers 2-4 mm long, elliptical or lanceolate; connective prolonged into an aristate awn $1-2 \mathrm{~mm}$ long; ovary ca. 4 mm long, short pubescent, ovate, with branched trichomes, rare simple trichomes, sessile; style $3-4 \mathrm{~mm}$ long, twisted or straight, apex entire. Fruits ellipsoid or orbicular; 4 (5) valves $2-4 \times 1.5-2.5 \mathrm{~cm}$, covered by bristles $1-2 \mathrm{~mm}$ long. Seeds 1, elliptical, ca. 10 x ca. $0.5 \mathrm{~mm}$, aril not seen.

Geographical distribution: Sloanea uniflora occurs in gallery forests, often at waterlogged sites or close to watercourses in the Brazilian states of Mato Grosso, Mato Grosso do Sul and Goiás.

\section{Acknowledgments}

We thank FAPESP (Fundação de Amparo à Pesquisa do Estado de São Paulo) for the $\mathrm{PhD}$ thesis research fellowship; to the KLARF (The Kew Latin American Research Fellowships Programme) for the fellowship at the Royal Botanic Gardens Kew; to Isabela Mascia Silveira and Erik Russel Wild for the English revision of the manuscript, and Rogério Lupo for the illustrations. We also wish to thank the anonymous reviewer of Acta Botanica Brasilica for the contribution to this work.

\section{References}

Boeira ASP, Vicentini A, Ribeiro JELS. 2012. Three new species of Sloanea (Elaeocarpaceae) from de Central Amazon, Brazil. Brazilian Journal of Botany 35: 119-123.

Brummit RK, Powell CE. 1992. Authors of plant names. Royal Botanic Gardens, Kew.

Coode MJE. 1983. A conspectus of Sloanea (Elaeocarpaceae) in the Old World. Kew Bulletin 38: 347-427.

Hickey LJ. 1979. Classification of the architecture of dicotyledonous leaves. American Journal of Botany 60: 17-33.

Holmgren PK, Holmgren NH. 1998. Onwards (continuously updated). Index Herbariorum. New York: Botanical Garden. <http://sciweb. nybg.org/Science2/IndexHerbariorum.asp >. 12 Jan. 2015.

Palacios-Duque L. 2004. Dos nuevas especies de Sloanea (Elaeocarpaceae) Del Chocó, Colombia. Caldasia 2: 429-432.

Palacios-Duque L. 2005. Sloanea pacuritana Pal.- Duque sp. nov. (Elaeocarpaceae) del Chocó (Colombia). Anales del Jardim Botanico de Madrid 62: 191-193.

Palacios-Duque L. 2007. Sloanea esmeraldana Pal.-Duque sp. nov. (Elaeocarpaceae), del Chocó (Colombia). Anales del Jardim Botanico de Madrid 64: 103-105.

Palacios-Duque L, Fernándes-Alonso JL. 2005. Uma nueva e interesante especie de Sloanea (Elaeocarpaceae) del Pacifico Colombiano. Revista de La Academia Colombiana de Ciencias Exactas, Fisicas y Naturales 29: 179-182.

Radford AE, Dickison WC, Massey JR, Bell CR.1976. Vascular Plant Systematics. New York, Harper and Row. 
Sampaio D. 2009. Revisão taxonômica das espécies neotropicais extraamazônicas de Sloanea L. (Elaeocarpaceae) na América do Sul. PhD Thesis, Universidade Estadual de Campinas, Brazil.

Sampaio D, Souza VC. 2010. New species of Sloanea (Elaeocarpaceae) from the Brazilian Cerrado. Rodriguesia 61: 13-15.

Sampaio D, Souza VC. 2011a. Two new species of Sloanea (Elaeocarpaceae) from the Brazilian Atlantic Forest. Kew Bulletin 66: 511-515.

Sampaio D, Souza VC. 2011b. Three new species of Sloanea (Elaeocarpaceae). Phytotaxa 16: 45-51.

Sampaio D, Souza VC. 2014. Typification of some species of Sloanea (Elaeocarpaceae). Phytotaxa 184: 121-130.

Schumann KM. 1886. Tiliaceae. In: Martius CFP. (ed.) Flora Brasiliensis. Vol. 12(3). Munich and Leipzig, R. Oldenbourg. p. 117-200.

Smith CE. 1954 The new world species of Sloanea (Elaeocarpaceae). Contributionsfrom the Gray Herbarium of Harvard University 175: $1-144$.
Smith CE. 1965. Elaeocarpaceae. In: Woodson RE, Schery RW. (eds.) Flora of Panama. Annals of the Missouri Botanical Garden 52: 487-495.

Smith CE.1967. Elaeocarpaceae. In: Steyermark JA. (ed.) Flora del AuyanTepui. Vol. 2. Caracas, Acta Botanica Venezuelica 2: 244-246.

Smith DA. 1996. Three previously undescribed Central American species of Sloanea (Elaeocarpaceae). Novon 6: 120-127.

Stafleu FA, Cowan RS. 1981. Taxonomic Literature, 2nd. edn. Utrecht, Bohn, Scheltema and Holkema.

Steyermark JA. 1952. Botanical Exploration in Venezuela- II. Fieldiana: Bot. 28: 357-363.

Steyermark JA. 1988. Flora of the Venezuelan Guayana-VI. Annals of the Missouri Botanical Garden 75: 1565-1586.

Steyermark JA. 1978. Novedades venezolanas del genero Sloanea. Pittieria 7: 13-16.

Weberling FHE. 1989. Morphology of flowers and inflorescences; translated by R. J. Pankhurst. Cambridge and New York, Cambridge University Press. 